\title{
Artroplastia reversa de ombro: Avaliação dos resultados clínicos e funcionais de acordo com a etiologia
}

\section{Reverse Shoulder Arthroplasty: Evaluation of the Clinical and Functional Outcomes per Etiology}

\author{
Thiago Medeiros Storti ${ }^{1,2(0)}$ Thiago da Silva Ribeiro $^{3(0)}$ Rafael Salomon Silva Faria1(1) \\ João Eduardo Simionatto ${ }^{10}$ Carolina Simionatto ${ }^{10}$ Alexandre Firmino Paniago ${ }^{1,2}$ (잉
}

1 Instituto do Ombro de Brasília, Brasília, DF, Brasil

${ }^{2}$ Hospital Ortopédico e Medicina Especializada (HOME), Instituto de Pesquisa e Ensino (IPE-HOME), Brasília, DF, Brasil

3 Instituto do Ombro de Brasília, Brasília, DF, Brasil
Endereço para correspondência Thiago Medeiros Storti, Hospital Ortopédico e Medicina Especializada (HOME), SGAS Quadra 613 - Conjunto C - Asa Sul Brasília, DF, Brasil (e-mail: thiago_storti@hotmail.com).

Rev Bras Ortop 2022;57(5):868-875.

\begin{abstract}
Resumo
Palavras-chave

- artropatias

- manguito rotador

- ruptura

- artroplastia do ombro

- dor de ombro

- lesões do ombro

- prótese de ombro

Objetivo Avaliar os resultados clínicos da artroplastia reversa do ombro no tratamento de suas diversas indicações.

Métodos Estudo longitudinal retrospectivo que analisou os resultados dos escores Constant, UCLA e amplitudes de movimentos dos pacientes submetidos à artroplastia reversa do ombro. Resultados Foram analisados 28 pacientes, a média de idade foi de 75.6 anos, com seguimento médio de 45 meses. No geral, obtivemos uma variação significativa ( $p<0,0001)$ entre o escore UCLA pré-operatório $(10,2$ pontos) e o escore UCLA pósoperatório ( 29,6 pontos), o que corresponde a um aumento relativo de aproximadamente $200 \%$. Além disso, obtivemos pontuação média do escore Constant de 67,8 e uma taxa de complicações de $17,8 \%$. Quanto aos resultados funcionais segundo as indicações, os casos de sequela de fratura apresentaram as melhores médias de elevação $\left(165^{\circ}\right)$, escore Constant (79 pontos), escore UCLA pós-operatório (32,5 pontos) e aumento absoluto na variação do escore UCLA (22 pontos), sem significância estatística. Porém, identificou-se que os casos operados por sequela de fratura apresentaram elevação $(p=0,027)$ e pontuação no escore Constant $(p=0,047)$ significativamente maiores em relação aos casos de artropatia do manguito rotador. Além disso, observamos que as menores médias dos escores Constant e UCLA pós-operatórios foram obtidos nas seguintes etiologias: artrose primária, fratura aguda e revisão de artroplastia.

Conclusão A artroplastia reversa de ombro apresentou resultados funcionais satisfatórios, podendo ser uma opção de tratamento não somente nos casos de artropatia do manguito rotador, mas também em várias outras patologias.
\end{abstract}

\footnotetext{
* Trabalho desenvolvido no Hospital Ortopédico e Medicina Especializada (HOME), Instituto de Pesquisa e Ensino (IPEHOME), Brasília, DF, Brasil.
}

recebido

27 de Setembro de 2020

aceito

11 de Fevereiro de 2021

Publicado on-line

de Janeiro 20, 2022
DOI https://doi.org/ $10.1055 / \mathrm{s}-0041-1731674$ ISSN 0102-3616.
(C) 2022. Sociedade Brasileira de Ortopedia e Traumatologia. All rights reserved.

This is an open access article published by Thieme under the terms of the Creative Commons Attribution-NonDerivative-NonCommercial-License, permitting copying and reproduction so long as the original work is given appropriate credit. Contents may not be used for commercial purposes, or adapted, remixed, transformed or built upon. (https://creativecommons.org/ licenses/by-nc-nd/4.0/)

Thieme Revinter Publicações Ltda., Rua do Matoso 170, Rio de Janeiro, RJ, CEP 20270-135, Brazil 


\begin{abstract}
Keywords

- arthropathy

- rotator cuff

- rupture

- arthroplasty, replacement, shoulder

- shoulder pain

- shoulder injuries

- shoulder prosthesis

Objective The present study aimed to evaluate the clinical outcomes of reverse shoulder arthroplasty to treat several conditions.

Methods Retrospective, longitudinal study analyzing the Constant and University of California at Los Angeles (UCLA) scores and range of motion of patients undergoing reverse shoulder arthroplasty.

Results In total, 28 patients were analyzed, with a mean age of 75.6 years old. The mean duration of follow-up was 45 months. Overall, there was a significant variation $(p<0.0001)$ between the preoperative (10.2 points) and the postoperative UCLA scores (29.6 points), corresponding to a relative increase of approximately $200 \%$. In addition, the mean Constant score was 67.8 , and the complication rate was $17.8 \%$. As for functional outcomes per etiology, fracture sequelae cases presented the best mean elevation $\left(165^{\circ}\right)$, Constant score (79 points), postoperative UCLA score (32.5 points), and absolute delta UCLA score increase (22 points), but with no statistical significance. However, cases operated for fracture sequelae showed significantly higher elevation $(p=0.027)$ and Constant score $(p=0.047)$ compared to rotator cuff arthropathy cases. In addition, the lowest mean postoperative Constant and UCLA scores were observed for the following etiologies: primary arthrosis, acute fracture, and arthroplasty revision.

Conclusion Reverse shoulder arthroplasty showed satisfactory functional outcomes and may be a treatment option not only for rotator cuff arthropathy but for several other conditions.
\end{abstract}

\section{Introdução}

A artroplastia reversa do ombro (ARO) foi desenvolvida em 1985 por Grammont et al. ${ }^{1}$ Seu princípio se baseia na medialização e inferiorização do centro de rotação da articulação glenoumeral, mecanismo que aumenta o braço de alavanca e o momento de força do deltoide, compensando o manguito rotador (MR) insuficiente. ${ }^{1,2}$

Além da artropatia do manguito rotador (AMR), ${ }^{3-5}$ atualmente suas indicações incluem lesões extensas do $M R^{3,5,6}$ sequelas de fraturas, ${ }^{3,7}$ artropatias inflamatórias, ${ }^{3}$ fraturas graves da extremidade proximal do úmero, ${ }^{3,5}$ revisão de artroplastias primárias ${ }^{3,5}$ e reconstrução após ressecção tumoral. $^{3,5}$ Este procedimento é indicado principalmente aos pacientes idosos com função do ombro diminuída e elevação anterior ativa menor que $90^{\circ}, 3$ sendo contraindicado caso haja ausência ou comprometimento grave da contração do deltoide. ${ }^{3}$

As complicações, cujas taxas variam de 7,8 a 24\%, incluem notching escapular, infecção, instabilidade, hematoma, soltura de componentes, fraturas periprotéticas, e lesões neurovasculares. ${ }^{8-10}$

A artroplastia reversa de ombro é, portanto, um procedimento muito útil para o tratamento de diversas patologias complexas do ombro e seus resultados e complicações em cada uma dessas vem sendo cada vez mais estudados. 0 objetivo do nosso trabalho é avaliar o resultado funcional do uso da ARO no tratamento de diversas etiologias. Diante do exposto e baseado em estudos da literatura, ${ }^{10}$ hipotetizamos que os pacientes com diagnóstico de artropatia do manguito rotador apresentarão melhores resultados quando submeti- dos à artroplastia reversa quando comparados aos pacientes com outras etiologias.

\section{Metodologia}

\section{Design do Estudo e Participantes}

Trata-se de um estudo longitudinal retrospectivo. Durante o período de 2011 a 2016, 35 pacientes foram submetidos à ARO. Foram incluídos no estudo os pacientes com o período mínimo de 24 meses de acompanhamento. Foram excluídos aqueles que não aceitaram participar da pesquisa e aqueles que não conseguimos contactar. Após a aplicação dos critérios (inclusão e exclusão), 28 pacientes aceitaram participar da pesquisa e comparecer ao hospital para serem reavaliados, totalizando 28 ombros.

\section{Aprovações Éticas}

Todos os pacientes assinaram o termo de consentimento livre esclarecido. Este estudo foi submetido à avaliação e à aprovação do Comitê de Ética e Pesquisa com Seres Humanos, com número do parecer 2.430.846, CAAE: 70804417.0.0000.0023.

Procedimento Cirúrgico e Reabilitação Pós-operatória Todos os procedimentos foram realizados por um de três cirurgiões. As próteses implantadas foram Delta Xtend (DePuy Synthes, Warsaw, IN, EUA) ou Equinoxe Reverse Shoulder (Exactech, Inc., Gainesville, FL, EUA) ( - Figura 1).

$\mathrm{O}$ acesso deltopeitoral foi o escolhido em todos os pacientes. O número de parafusos utilizados para fixar a placa base foi determinado pelo estoque ósseo da glenoide e pela preferência do cirurgião. Nos casos em que o tendão do 

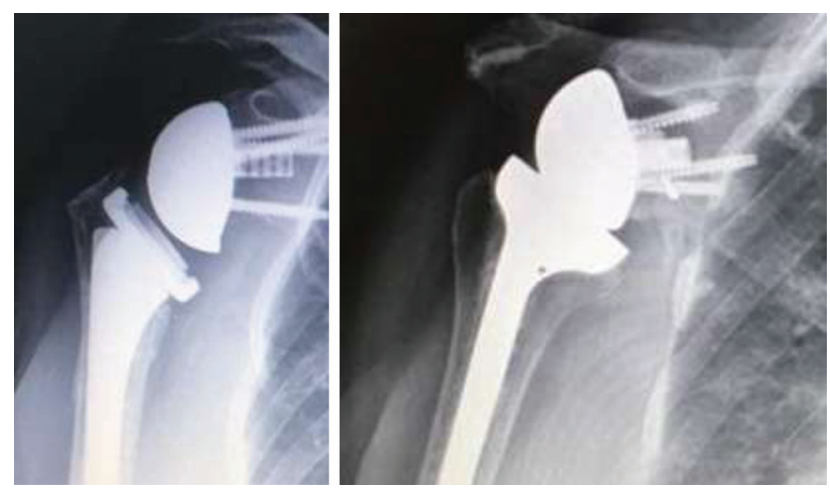

Fig. 1 Os dois modelos de prótese utilizados em nossos casos (Equinoxe Reverse Shoulder à esquerda e Delta Xtend à direita).

músculo subescapular estava íntegro, foi realizada sua desinserção do tubérculo menor e posterior fixação através de pontos transósseos com suturas não absorvíveis.

Quanto à reabilitação pós-operatória, todos os pacientes seguiram o mesmo protocolo padronizado. Eles permaneceram imobilizados com tipoia por 6 semanas, iniciando, após esse período e sob supervisão de fisioterapeutas, o ganho de amplitude de movimento (ADM), inicialmente passivos e ativos-assistidos com limitação de $90^{\circ}$ para abdução e flexão frontal. $\mathrm{O}$ fortalecimento muscular foi estimulado a partir do $3^{\circ}$ mês pós-operatório, sendo desaconselhada a realização de carga no membro antes desse período.

\section{Desfechos de análise}

A coleta de dados durante o seguimento pós-operatório foi realizada em duas etapas: 1 - consulta aos prontuários para coleta de características demográficas, cirúrgicas e funcionais; e 2 - avaliação clínica com coleta de variáveis funcionais e ADM.

A partir dos prontuários médicos, foram coletados os dados referentes à idade, tempo de seguimento, sexo, lateralidade do ombro operado, diagnóstico, presença de complicações e escore da Univerity of California at Los Angeles $(\text { UCLA })^{11}$ pré-operatório. Devido à impossibilidade de se aplicar o escore UCLA no pré-operatório aos pacientes com fratura aguda da extremidade proximal do úmero $(n=2)$, este escore não foi calculado previamente à cirurgia em dois pacientes.

O diagnóstico foi enquadrado em uma das seguintes afecções: artropatia do manguito rotador (AMR), artrose primária com diagnóstico associado de lesão do MR (pacientes idosos), fraturas agudas complexas com head split da extremidade proximal do úmero em pacientes idosos (-Figura 2), lesão extensa irreparável do MR com perda crônica da elevação do membro, revisão de artroplastia e sequela de fratura.

Foram consideradas como irreparáveis as lesões do MR que: acometeram mais de 1 tendão, ou lesões maiores de $5 \mathrm{~cm}$, e aquelas com degeneração gordurosa dos ventres musculares grau III ou IV, de acordo com o sistema de classificação descrito por Goutallier et al. ${ }^{12}$

A análise funcional do ombro foi realizada por pesquisador capacitado, que não compunha a equipe cirúrgica. Ao
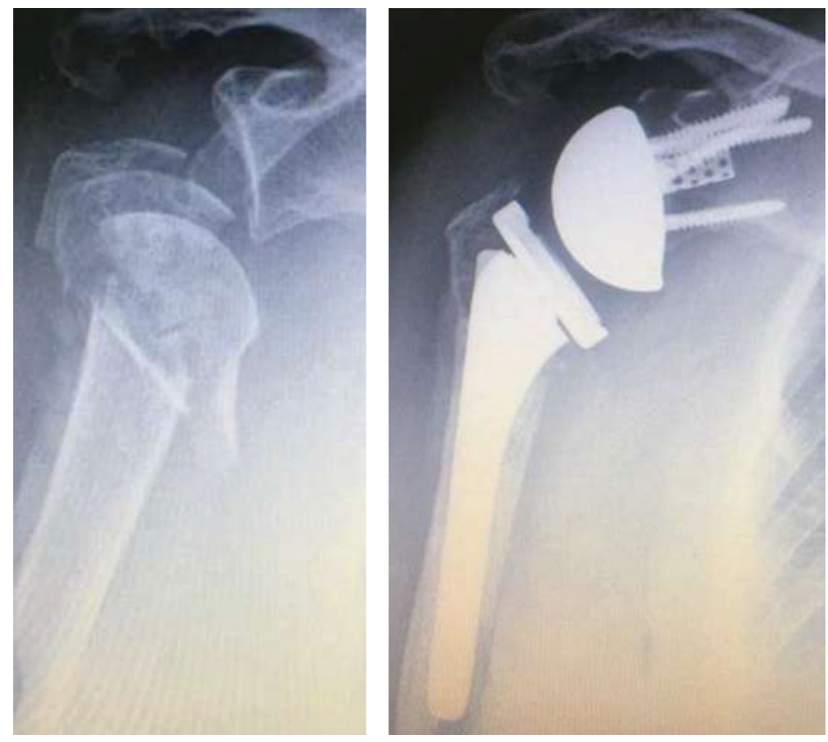

Fig. 2 Pré e pós-operatório de fratura aguda complexa com head split da extremidade proximal do úmero em paciente idoso.

seguimento, foram aplicados os escores Constant ${ }^{13}$ e UCLA, ${ }^{11}$ e foram aferidas por goniometria a ADM ativa de elevação, rotação lateral (RL) e rotação medial (RM).

Além disso, foi realizada a comparação entre os parâmetros de ADM, escores funcionais e grau de satisfação entre subgrupos segundo faixa etária, sexo e lateralidade; e foi verificado a associação entre tempo de acompanhamento e idade, ADM e escores funcionais.

A pesquisa de satisfação proposta pelo autor foi realizada questionando-se aos pacientes se estes estavam muito satisfeitos, satisfeitos ou insatisfeitos com os resultados obtidos.

\section{Estatística}

Após a tabulação dos dados, procedeu-se à análise descritiva, expressa pelas medidas de tendência central e de dispersão adequadas para os dados numéricos e pela frequência e percentual para os dados categóricos, bem como calculouse a diferença entre o escore UCLA pós e pré-operatórios (delta UCLA).

A análise estatística foi realizada por estatístico independente e foi composta pelo teste dos postos sinalizados de Wilcoxon para verificar a variação do escore UCLA do pré para pós-operatório, análise que não se aplica aos pacientes operados por fratura aguda; pelos testes t de Student, MannWhitney, qui-quadrado e exato de Fisher para a comparação entre os parâmetros de ADM, escores funcionais e grau de satisfação entre subgrupos segundo faixa etária, sexo e lateralidade; e pelo coeficiente de correlação de Spearman para verificar a associação entre tempo de acompanhamento e idade, ADM e escores funcionais.

Foi realizada uma análise prévia para verificar a normalidade das variáveis por meio do teste de Shapiro-Wilk juntamente com a análise gráfica dos histogramas. Para as variáveis que apresentaram distribuição não-Gaussiana, foi utilizado teste não paramétrico. $O$ critério de determinação de significância adotado foi ao nível de $5 \%$. A análise 
estatística foi processada pelo software estatístico SAS System, versão 6.11 (SAS Institute, Inc., Cary, North Carolina, EUA).

Para verificar se existia diferença significativa nos parâmetros avaliados em relação às etiologias estudadas, realizou-se uma análise extremamente exploratória. Fica claro que esta análise foi feita em caráter exploratório devido ao número muito reduzido de algumas categorias $(n=2)$; por este motivo, o intervalo intrquartil (IIQ) não possui o limite superior (Q3).

Inicialmente, a análise de variância (ANOVA) de KruskalWallis foi aplicada para saber se existia diferença significativa, ao nível de $5 \%$, nas variáveis comparando as seis patologias simultaneamente; e o teste de Mann-Whitney (MW) foi usado, apenas em caráter exploratório, para identificar as tendências dos resultados.

\section{Resultados}

\section{Resultados Clínicos e Funcionais Gerais}

Foram avaliados 28 pacientes. A média de idade foi de 75.6 anos (variou de 58-86 anos). 0 período médio de seguimento foi de 45 meses (variou de 24-84 meses). A amostra foi composta por $89.3 \%$ de pacientes do sexo feminino. 0 ombro mais frequentemente acometido foi o direito, correspondendo a 75\% dos casos (- Tabela 1).

Tabela 1 Caracterização clínica da amostra

\begin{tabular}{|c|c|c|}
\hline Variável & & \\
\hline \multicolumn{3}{|l|}{ Idade (anos) } \\
\hline $\begin{array}{l}\text { média } \pm \text { DP } \\
\text { (mínimo - máxima) }\end{array}$ & \multicolumn{2}{|c|}{$75,6 \pm 6,4(58-86)$} \\
\hline Idade à cirurgia & $\mathrm{N}$ & $\%$ \\
\hline$>75$ anos & 15 & 53,6 \\
\hline$\leq 75$ anos & 13 & 46,4 \\
\hline \multicolumn{3}{|l|}{ Sexo } \\
\hline masculino & 3 & 10,7 \\
\hline feminino & 25 & 89,3 \\
\hline \multicolumn{3}{|l|}{ Seguimento (meses) } \\
\hline $\begin{array}{l}\text { média } \pm \text { DP } \\
\text { (mínima-máxima) }\end{array}$ & \multicolumn{2}{|c|}{$45 \pm 16,7(24-84)$} \\
\hline \multicolumn{3}{|l|}{ Ombro } \\
\hline direito & 21 & 75,0 \\
\hline esquerdo & 7 & 25,0 \\
\hline \multicolumn{3}{|l|}{ Doença } \\
\hline $\begin{array}{l}\text { Artropatia do } \\
\text { manguito rotador }\end{array}$ & 11 & 39,3 \\
\hline Artrose primária & 2 & 7,1 \\
\hline Fratura aguda & 2 & 7,1 \\
\hline $\begin{array}{l}\text { Lesão extensa do } \\
\text { manguito rotador }\end{array}$ & 8 & 28,6 \\
\hline Revisão de prótese & 3 & 10,8 \\
\hline Sequela de fratura & 2 & 7,1 \\
\hline
\end{tabular}

Abreviatura: DP, desvio padrão.
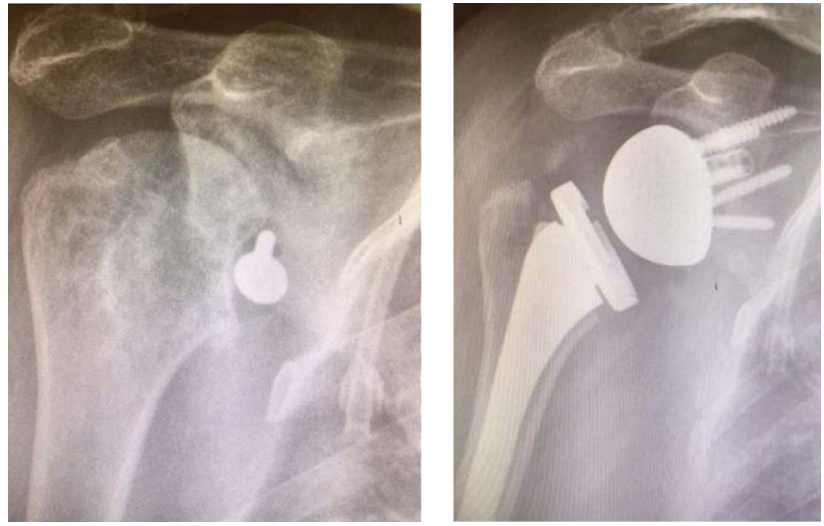

Fig. 3 Pré e pós-operatório do grupo sequela de fratura.

Dentre as indicações para o procedimento, a mais prevalente foi a AMR (39.3\%), seguida pela lesão extensa irreparável do MR (28.6\%) e revisão de artroplastia (10.8\%). As demais indicações corresponderam, cada uma, a 7.1\% dos casos (-Tabela 1). Os dois pacientes com diagnóstico de sequela de fratura apresentavam: (1) fratura da porção anterior da glenoide com instabilidade anterior do ombro, que evoluiu com subluxação anterior da cabeça umeral e artropatia secundária ao implante (-Figura 3) e (2) fratura complexa do úmero proximal há 5 anos tratada de forma não cirúrgica, evoluindo com artrose gleno-umeral sintomática. Já os pacientes do grupo de revisão de artroplastia haviam sido submetidos previamente a artroplastia parcial (1 paciente) ou total anatômica (1 paciente).

Os valores médios encontrados para a ADM ativa foram de $137.6^{\circ}$ para elevação, $16.7^{\circ}$ para $R L$, e entre L1 e L2 para RM (-Tabela 2).

Em relação aos escores funcionais, o escore de UCLA préoperatório $(n=26)$ apresentou uma mediana de 10 pontos, $\mathrm{e}$ o UCLA pós-operatório $(n=26)$ teve seu valor mediano de 31 pontos, variação significativa ( $p<0,0001$ ), o que corresponde a um aumento relativo de aproximadamente $200 \%(n=26)$, conforme ilustra a - Figura 4.

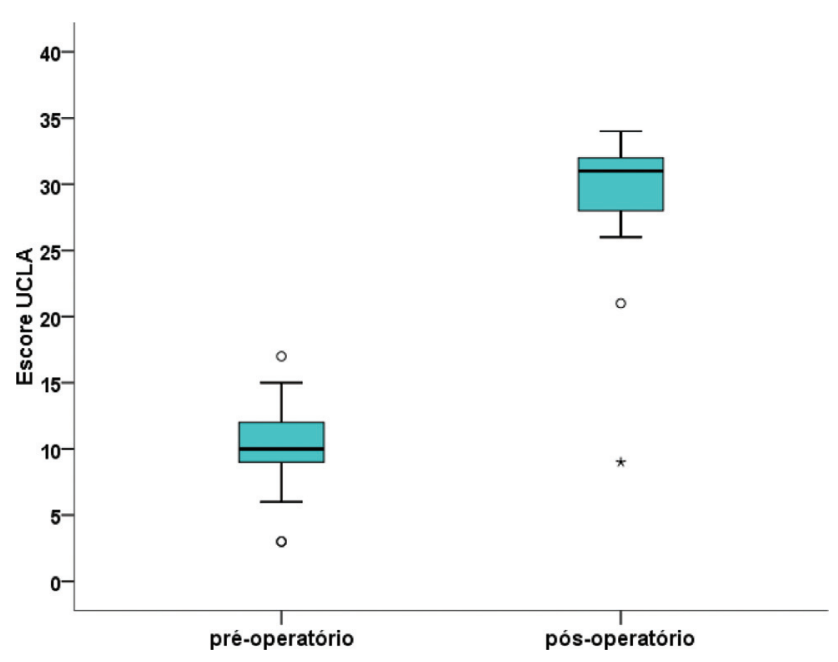

Fig. 4 Escore UCLA no pré e pós-operatório. 
Tabela 2 Escore funcional e grau de satisfação

\begin{tabular}{|c|c|c|}
\hline Variável & & \\
\hline \multicolumn{3}{|l|}{ Escore Constant } \\
\hline $\begin{array}{l}\text { média } \pm \text { DP } \\
\text { (mínima-máxima) }\end{array}$ & \multicolumn{2}{|c|}{$67,8 \pm 12(28-93)$} \\
\hline \multicolumn{3}{|l|}{ Escore UCLA pré-op * } \\
\hline $\begin{array}{l}\text { média } \pm \mathrm{DP} \\
\text { (mínima-máxima) }\end{array}$ & \multicolumn{2}{|c|}{$10,2 \pm 3,2(3-17)$} \\
\hline \multicolumn{3}{|l|}{ Escore UCLA pós-op * } \\
\hline $\begin{array}{l}\text { média } \pm \text { DP } \\
\text { (mínima-máxima) }\end{array}$ & \multicolumn{2}{|c|}{$29,6 \pm 5,1(9-34)$} \\
\hline \multicolumn{3}{|l|}{ Delta absoluto UCLA } \\
\hline $\begin{array}{l}\text { média } \pm \text { DP } \\
\text { (mínima-máxima) }\end{array}$ & \multicolumn{2}{|c|}{$19,4 \pm 5(6-31)$} \\
\hline \multicolumn{3}{|l|}{ Delta (\%) UCLA } \\
\hline $\begin{array}{l}\text { média } \pm \text { DP } \\
\text { (mínima-máxima) }\end{array}$ & \multicolumn{2}{|c|}{$228 \pm 180,2(86,7-1.033)$} \\
\hline \multicolumn{3}{|l|}{ Elevação $\left({ }^{\circ}\right)$} \\
\hline $\begin{array}{l}\text { média } \pm \text { DP } \\
\text { (mínima-máxima) }\end{array}$ & \multicolumn{2}{|c|}{$137,6 \pm 25,1(50-165)$} \\
\hline \multicolumn{3}{|l|}{ Rotação lateral } \\
\hline $\begin{array}{l}\text { média } \pm \text { DP } \\
\text { (mínima-máxima) }\end{array}$ & \multicolumn{2}{|c|}{$16,7 \pm 17,7(0-60)$} \\
\hline \multicolumn{3}{|c|}{ Rotação medial (vértebra lombar) } \\
\hline média & \multicolumn{2}{|c|}{$\mathrm{L} 1 / \mathrm{L} 2$} \\
\hline \multicolumn{3}{|l|}{ Grau de satisfação } \\
\hline insatisfeito & 1 & 3,6 \\
\hline satisfeito & 11 & 39,3 \\
\hline muito satisfeito & 16 & 57,1 \\
\hline
\end{tabular}

Abreviatura: DP, desvio padrão.

* Não se aplica a dois pacientes $(n=26)$.

O outro escore funcional avaliado foi o de Constant, pósoperatório, com média de 69.4 pontos (-Tabela 2 ).

Quanto à avaliação de satisfação, apenas uma paciente declarou-se insatisfeita com os resultados, os demais declararam-se satisfeitos (39.3\%) ou muito satisfeitos (57.1\%) (-Tabela 2). Essa paciente, de 75 anos, - cuja indicação cirúrgica foi a revisão de hemiartroplastia realizada em fratura complexa da extremidade proximal do úmero que evoluiu com ruptura do MR, dor intensa e redução importante da ADM - apresentou UCLA pré-artroplastia reversa de 3 pontos. Na avaliação funcional realizada 24 meses após a ARO, a paciente apresentou os seguintes resultados: $50^{\circ}$ de elevação anterior, $0^{\circ}$ de RL, RM ao nível do glúteo, 28 pontos no escore Constant e 9 pontos no escore UCLA (- Figura 5).

\section{Resultados clínicos e funcionais de acordo com a etiologia}

Quanto aos resultados funcionais segundo a etiologia, observou-se que a indicação sequela de fratura apresentou os melhores resultados para elevação, RL, Constant, UCLA pósoperatório e aumento absoluto na variação do escore UCLA

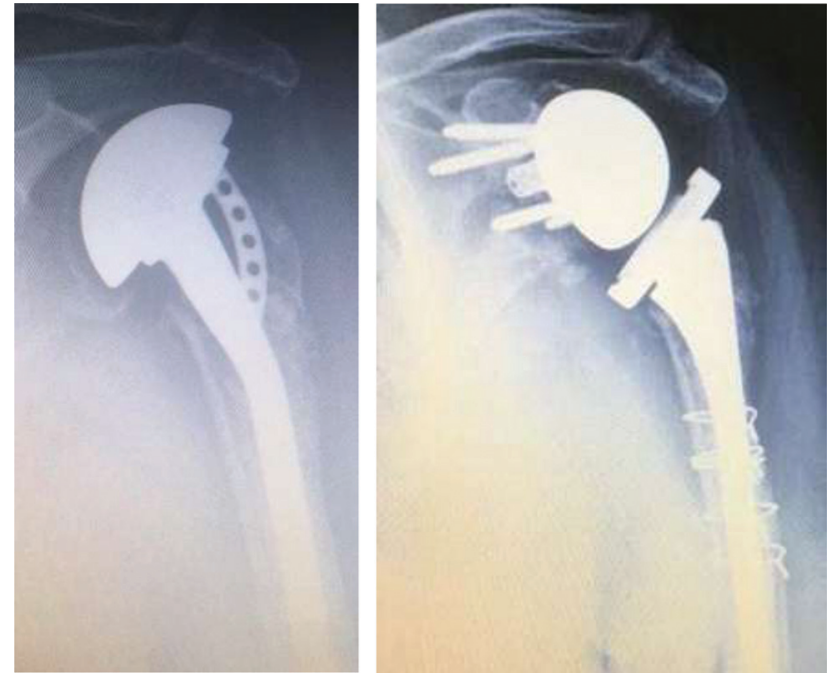

Fig. 5 Pré e pós-operatório do grupo revisão de artroplastia primária.

(-Tabela 3). Já a revisão de prótese foi a indicação com os piores resultados para elevação, RL, Constant, UCLA pósoperatório e variação do escore UCLA (tabela 3). Segundo o teste de MW, identificou-se que os casos operados por sequela de fratura apresentaram elevação $(p=0,027)$ e pontuação no escore Constant $(p=0,047)$ significativamente maiores em relação aos casos de AMR (-Tabela 4). Já os pacientes submetidos a revisão de artroplastia prévia apresentaram RL significativamente menor em relação aos pacientes operados por AMR, fratura aguda, lesões extensas do MR e sequela de fratura (tabela 4).

\section{Complicações}

Complicações pós-operatórias foram observadas em 5 pacientes (17,8\%): (1) 1 caso de fratura periprotética do úmero durante o procedimento cirúrgico, distal à extremidade da haste, sendo tratado de maneira não cirúrgica, evoluindo com completa consolidação; (2) outro caso de fratura periprotética, ocorrida por queda 19 meses após a artroplastia reversa, sendo tratada com osteossíntese e fixação com placa e parafusos ( - Figura 6); (3) 1 caso de infecção tratada com desbridamento cirúrgico e antibioticoterapia; (4) 1 caso de neuropraxia do nervo radial, com completa recuperação em 3 meses; (5) 1 caso de luxação da prótese 2 meses após a ARO, tratada com redução sob anestesia, sem necessidade de novo procedimento.

A fratura periprotética transoperatória e a neuropraxia do radial ocorreram no grupo de AMR. A infecção foi identificada em um dos casos de artrose primária, e a fratura periprotética tardia ocorreu no grupo de fratura aguda da extremidade proximal do úmero. Além disso, a luxação da prótese se deu em um dos casos de revisão de artroplastia.

\section{Discussão}

Atualmente a AMR é a indicação mais frequente de ARO, enquanto que a prevalência das demais indicações varia de acordo com o estudo analisado. ${ }^{4,5,14,15}$ Em nosso estudo, entre as indicações para o procedimento, a mais comum foi a 
Tabela 3 Variável clínica, escore funcional e parâmetro ADM segundo a etiologia

\begin{tabular}{|c|c|c|c|c|c|c|c|c|c|c|c|c|}
\hline \multirow[t]{2}{*}{ Variável } & \multicolumn{2}{|c|}{ AMR } & \multicolumn{2}{|c|}{$\begin{array}{l}\text { Artrose } \\
\text { primária }\end{array}$} & \multicolumn{2}{|c|}{$\begin{array}{l}\text { Fratura } \\
\text { aguda }\end{array}$} & \multicolumn{2}{|c|}{$\begin{array}{l}\text { Lesão ex- } \\
\text { tensa do MR }\end{array}$} & \multicolumn{2}{|c|}{$\begin{array}{l}\text { Revisão de } \\
\text { artroplastia }\end{array}$} & \multicolumn{2}{|c|}{$\begin{array}{l}\text { Sequela de } \\
\text { fratura }\end{array}$} \\
\hline & $\mathbf{n}$ & média & $\mathrm{n}$ & média & $\mathrm{n}$ & média & $\mathbf{n}$ & média & $\mathrm{n}$ & média & $\mathbf{n}$ & média \\
\hline Clínica & 11 & & 2 & & 2 & & 8 & & 3 & & 2 & \\
\hline Idade à cirurgia (anos) & & 75,6 & & 79 & & 78,5 & & 75,3 & & 80 & & 64,5 \\
\hline Seguimento (meses) & & 45,5 & & 53 & & 25 & & 46,5 & & 33,3 & & 65 \\
\hline \multicolumn{13}{|l|}{ ADM } \\
\hline Elevação $\left({ }^{\circ}\right)$ & & 139,9 & & 135 & & 110 & & 150,6 & & 96,7 & & 165 \\
\hline Rotação lateral $\left({ }^{\circ}\right)$ & & 19,5 & & 0 & & 10 & & 24 & & 0 & & 20 \\
\hline $\begin{array}{l}\text { Rotação medial } \\
\text { (vértebra lombar) }\end{array}$ & & 13,5 & & 17 & & 16 & & 14 & & 15,5 & & 14 \\
\hline \multicolumn{13}{|l|}{ Funcional } \\
\hline Escore Constant & & 69,5 & & 61,5 & & 59 & & 73,4 & & 49 & & 79 \\
\hline Escore UCLA pré-operatório & & 10,5 & & 10,5 & & - & & 11,1 & & 6 & & 10,5 \\
\hline Escore UCLA pós-operatório & & 31,1 & & 28 & & 30 & & 30,6 & & 20,7 & & 32,5 \\
\hline Delta UCLA absoluto & & 20,5 & & 17,5 & & - & & 19,5 & & 14,7 & & 22 \\
\hline
\end{tabular}

Abreviaturas: AMR, artropatia do manguito rotador; MR, manguito rotador.

AMR (39.3\%), seguida pela lesão extensa do MR (28.6\%) e revisão de prótese (10.8\%). As demais indicações - artrose primária, fratura aguda e sequela de fratura - corresponderam, cada uma, a $7,1 \%$ dos casos.

A análise do tipo de indicação é importante porque a etiologia se relaciona com a taxa de sucesso e incidência de complicações. ${ }^{5}$ Em nossa análise, os casos de sequela de fratura apresentaram as melhores médias de elevação $\left(165^{\circ}\right)$, escore Constant (79 pontos), escore UCLA pós-operatório (32,5 pontos) e aumento absoluto na variação do escore UCLA (22 pontos), sem significância estatística. Porém, identificou-se que os casos operados por sequela de fratura apresentaram elevação $(p=0,027)$ e pontuação no escore Constant $(p=0,047)$ significativamente maior em relação aos casos de AMR.
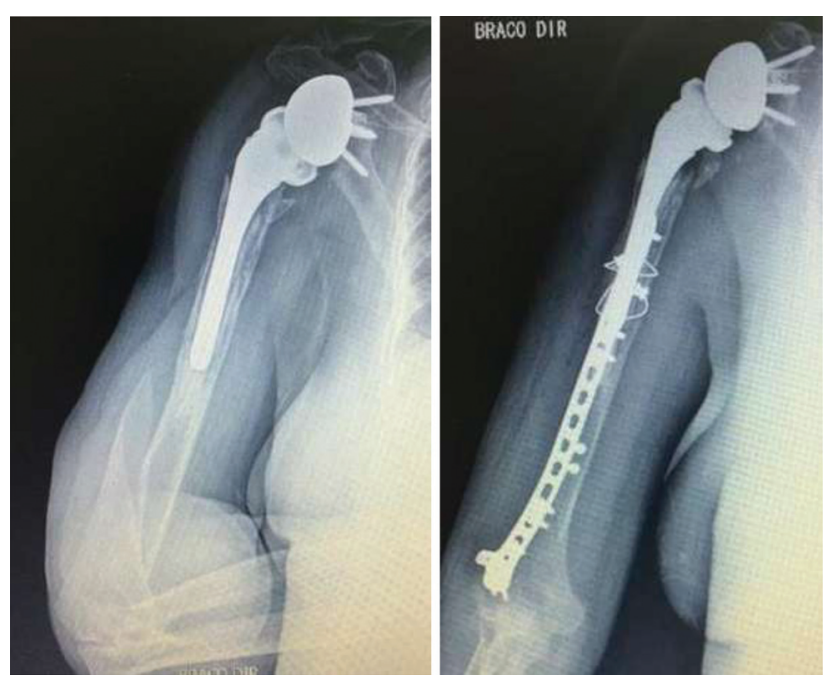

Fig. 6 Fratura periprotética tratada com osteossíntese com placa e parafusos.
Wall et al., ${ }^{16}$ em sua avaliação retrospectiva de 191 pacientes submetidos à artroplastia reversa por diversas etiologias, com média de seguimento de 39,9 meses, mostraram que os pacientes com AMR, artrose primária e lesão extensa do MR tiveram melhores resultados em relação aos pacientes que apresentavam como etiologia a artrose póstraumática e revisão de artroplastia. Semelhantemente, Wellmann et al. ${ }^{17}$ mostraram que os resultados da artroplastia reversa por AMR mostraram valores do escore de Constant significativamente maiores em relação aos pacientes com revisão de artroplastia.

Embora os pacientes do grupo revisão de prótese tenham apresentado uma melhora funcional no escore UCLA de magnitude similar ao dos outros grupos de etiologias, eles não atingiram o nível de desempenho observado entre os pacientes das outras etiologias. Wall et al. ${ }^{16}$ também obtiveram piores resultados funcionais para os pacientes de revisão de artroplastia, com $118^{\circ}$ de elevação do ombro e 52,2 pontos no escores Constant. Boileau et al. ${ }^{18}$ compararam os resultados da artroplastia reversa entre pacientes que apresentaram como etiologias AMR, sequela de fratura e revisão de artroplastia e também concluíram que os três grupos apresentaram ganho significativo na elevação e no escore de Constant, porém esses valores foram significativamente menores no grupo de revisão de artroplastia.

Apesar da amostra dos pacientes com AMR como etiologia não ter obtido os melhores resultados para os escores UCLA e Constant em nosso estudo, ao se analisar de forma isolada os resultados dessa indicação, observa-se que eles são semelhantes aos apresentados na maioria dos estudos analisados. ${ }^{5,16,17}$ Com isso, ratifica-se as informações contidas na literatura, que apresentam a AMR como uma indicação clássica com resultados funcionais satisfatórios. 


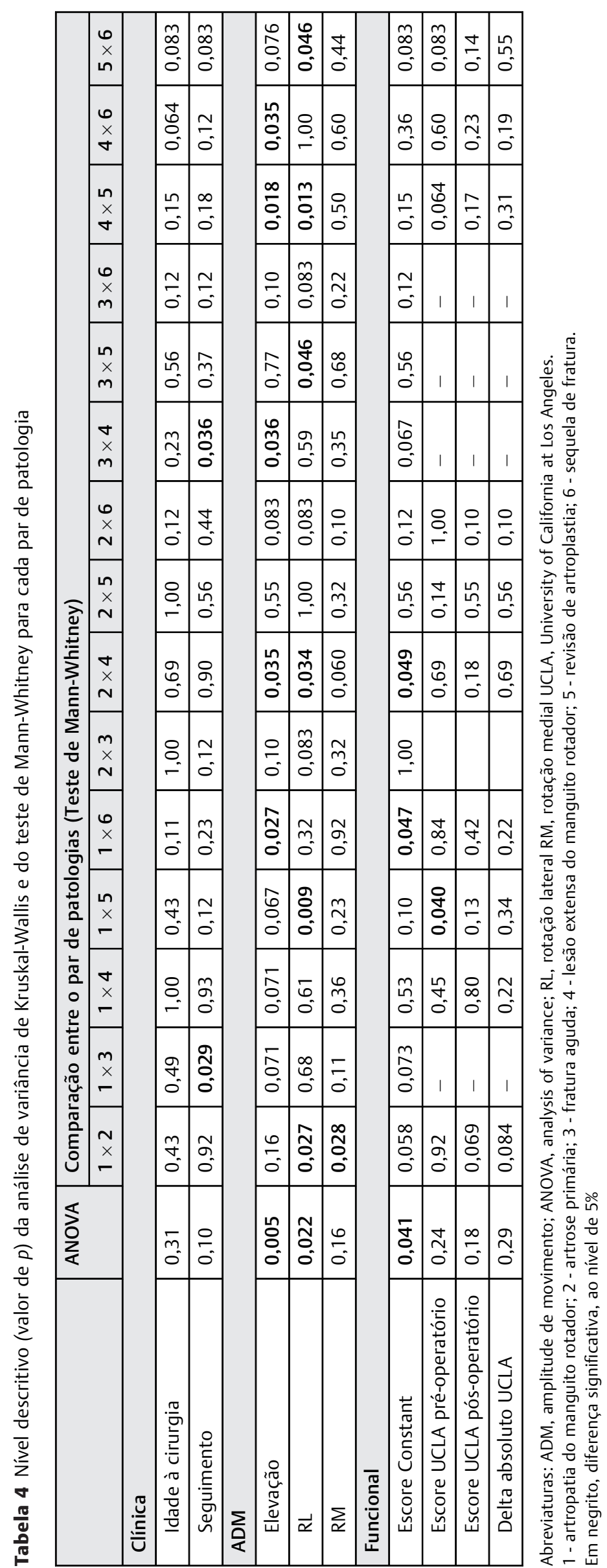


Fávaro et al., ${ }^{8}$ ao avaliar os resultados da artroplastia reversa em casos operados por artropatia do manguito, obtiveram uma melhora significativa no escore UCLA pré-operatório $(10,1) \mathrm{em}$ relação ao pós-operatório $(29,8)$. Esse resultado foi semelhante ao encontrado em nosso estudo, em que encontramos valor médio do escore UCLA pré-operatório de 10,2, mostrando diferença significativa $(p<0,0001)$ em relação ao valor médio do escore UCLA pós-operatório (29,6). O delta UCLA absoluto mostrou aumento médio de 19,4 pontos.

Outro escore funcional avaliado em nosso trabalho foi o de Constant pós-operatório, em que obtivemos valor médio de 67,8 pontos. Atalar et al. ${ }^{19}$ obtiveram média do escore Constant variando de 20.7 antes do procedimento para 58.9 ao final do seguimento. Já Amaral et al., ${ }^{20}$ ao avaliar os resultados da artroplastia reversa em casos de AMR, obtiveram média do escore de Constant de 60 pontos.

Quanto à ADM, nosso levantamento encontrou o valor médio de $137.6^{\circ}$ de elevação, inferior aos valores encontrados por Atalar et al. ${ }^{19}$ e Amaral et al., ${ }^{20}$ de $150^{\circ}$ e $149^{\circ}$, respectivamente. Em comparação aos mesmos autores, a média da RL de nossos pacientes foi de $16.7^{\circ}$, discretamente inferior aos mesmos, que mostraram média de $20^{\circ}$ e $37^{\circ}$, respectivamente. ${ }^{19,20}$ Essa diferença deve-se, possivelmente, ao fato de que ambos os trabalhos avaliaram apenas pacientes operados por AMR e com médias de idade inferiores à dos nossos pacientes. No entanto, a RM média em nossa amostra foi entre L1 e L2, superior à média encontrada nos mesmos estudos. 19,20

O índice de complicações encontrado em nosso estudo foi de $17,8 \%$ (5 casos). Wall et al. ${ }^{16}$ obtiveram incidência de $19,1 \%$ de complicações, sendo as mais frequentes a luxação (7,5\%) e a infecção (4\%), assim como Boileau et al., ${ }^{18}$ que obtiveram $24 \%$ de complicações pós-operatórias de artroplastia reversa, sendo também as mais comuns a luxação e a infecção.

Dentre as limitações do presente estudo estão a natureza observacional, a casuística pequena e a desproporção do número de pacientes entre os grupos de etiologias, dificultando a comparação entre os seus resultados. Quanto aos pontos fortes, destaca-se a análise pós-operatória realizada com diversas variáveis de funcionalidade do ombro.

\section{Conclusão}

A artroplastia reversa do ombro apresentou resultados funcionais satisfatórios, podendo ser uma opção de tratamento não somente nos casos de artropatia do manguito rotador, mas também em várias outras patologias. A etiologia préoperatória influencia os resultados clínicos da artroplastia reversa, com os pacientes submetidos à revisão de artroplastia apresentando resultados inferiores quando comparados aos pacientes com outras indicações.

\section{Suporte Financeiro}

Não houve suporte financeiro de fontes públicas, comerciais, ou sem fins lucrativos.

Conflito de Interesses

Os autores declaram não haver conflito de interesses.

\section{Referências}

1 Baulot E, Sirveaux F, Boileau P. Grammont's idea: The story of Paul Grammont's functional surgery concept and the development of the reverse principle. Clin Orthop Relat Res 2011;469(09):2425-2431

2 Rugg CM, Coughlan MJ, Lansdown DA. Reverse Total Shoulder Arthroplasty: Biomechanics and Indications. Curr Rev Musculoskelet Med 2019;12(04):542-553

3 Jazayeri R, Kwon YW. Evolution of the reverse total shoulder prosthesis. Bull NYU Hosp Jt Dis 2011;69(01):50-55

4 Ferreira Neto AA, Malavolta EA, Assunção JH, Trindade EM, Gracitelli MEC. Reverse shoulder arthroplasty: clinical results and quality of life evaluation. Rev Bras Ortop 2017;52(03):298-302

5 Samitier G, Alentorn-Geli E, Torrens C, Wright TW. Reverse shoulder arthroplasty. Part 1: Systematic review of clinical and functional outcomes. Int J Shoulder Surg 2015;9(01):24-31

6 Amoo-Achampong K, Krill MK, Acheampong D, Nwachukwu BU, McCormick F. Evaluating strategies and outcomes following rotator cuff tears. Shoulder Elbow 2019;11(1, Suppl):4-18

7 Pastor MF, Kieckbusch M, Kaufmann M, Ettinger M, Wellmann M, Smith T. Reverse shoulder arthroplasty for fracture sequelae: Clinical outcome and prognostic factors. J Orthop Sci 2019;24 (02):237-242

8 Fávaro RC, Abdulahad M, Filho SM, Valério R, Superti MJ. Rotator cuff arthropathy: what functional results can be expected from reverse arthroplasty? Rev Bras Ortop 2015; 50(05):523-529

9 Kempton LB, Ankerson E, Wiater JM. A complication-based learning curve from 200 reverse shoulder arthroplasties. Clin Orthop Relat Res 2011;469(09):2496-2504

10 Zumstein MA, Pinedo M, Old J, Boileau P. Problems, complications, reoperations, and revisions in reverse total shoulder arthroplasty: a systematic review. J Shoulder Elbow Surg 2011;20(01):146-157

11 Amstutz HC, Sew Hoy AL, Clarke IC. UCLA anatomic total shoulder arthroplasty. Clin Orthop Relat Res 1981;(155):7-20

12 Goutallier D, Postel JM, Bernageau J, Lavau L, Voisin MC. Fatty muscle degeneration in cuff ruptures. Pre- and postoperative evaluation by CT scan. Clin Orthop Relat Res 1994;(304): 78-83

13 Constant CR, Murley AH. A clinical method of functional assessment of the shoulder. Clin Orthop Relat Res 1987;(214):160-164

14 Holton J, Yousri T, Arealis G, Levy O. The Role of Reverse Shoulder Arthroplasty in Management of Proximal Humerus Fractures with Fracture Sequelae: A Systematic Review of the Literature. Orthop Rev (Pavia) 2017;9(01):27-31

15 Guery J, Favard L, Sirveaux F, Oudet D, Mole D, Walch G. Reverse total shoulder arthroplasty. Survivorship analysis of eighty replacements followed for five to ten years. J Bone Joint Surg Am 2006; 88(08):1742-1747

16 Wall B, Nové-Josserand L, O'Connor DP, Edwards TB, Walch G. Reverse total shoulder arthroplasty: a review of results according to etiology. J Bone Joint Surg Am 2007;89(07):1476-1485

17 Wellmann M, Struck M, Pastor MF, Gettmann A, Windhagen H, Smith T. Short and midterm results of reverse shoulder arthroplasty according to the preoperative etiology. Arch Orthop Trauma Surg 2013;133(04):463-471

18 Boileau P, Watkinson D, Hatzidakis AM, Hovorka I. Neer Award 2005: The Grammont reverse shoulder prosthesis: results in cuff tear arthritis, fracture sequelae, and revision arthroplasty. J Shoulder Elbow Surg 2006;15(05):527-540

19 Atalar AC, Salduz A, Cil H, Sungur M, Celik D, Demirhan M. Reverse shoulder arthroplasty: radiological and clinical short-term results. Acta Orthop Traumatol Turc 2014;48(01):25-31

20 Amaral MV, de Faria JL, Siqueira G, et al. Reverse arthroplasty of the shoulder for treating rotator cuff arthropathy. Rev Bras Ortop 2014;49(03):279-285 